\title{
Effectiveness and safety of pulsatile intravenous insulin therapy for the improvement of respiratory quotient in Chinese patients with diabetes mellitus
}

\author{
NAN GU ${ }^{1}$, AIMEI DONG ${ }^{1}$, LEI GAO ${ }^{2}$, CHENYING XIE $^{1}$, PEIYI HOU ${ }^{1}$, WENBO WANG $^{3}$, \\ SAINAN ZHU ${ }^{4}, \mathrm{CHEN} \mathrm{YAO}^{4}, \mathrm{JUNQING} \mathrm{ZHANG}^{1}$ and XIAOHUI GUO ${ }^{1}$ \\ ${ }^{1}$ Department of Endocrinology, ${ }^{2}$ Institute of Clinical Pharmacology, Peking University First Hospital, \\ Beijing 100034; ${ }^{3}$ Department of Endocrinology, Peking University Shougang Hospital, Beijing 100144; \\ ${ }^{4}$ Department of Biostatistics, Peking University First Hospital, Beijing 100034, P.R. China
}

Received May 31, 2019; Accepted December 20, 2019

DOI: $10.3892 /$ etm.2020.8563

\begin{abstract}
Pulsatile intravenous insulin therapy (PIVIT) is a means of imitating naturally occurring insulin pulses artificially. It is thought to improve carbohydrate metabolism, which can be assessed using the respiratory quotient (RQ). The aim of this present study was to assess the efficacy and safety of PIVIT for the improvement of RQ in Chinese patients with diabetes mellitus (DM). This 12-week, multi-center, prospective, randomized, open-label, parallel-group study involved $110 \mathrm{DM}$ patients (both type 1 and type 2) whose RQ was $<0.8$. Of these, 53 patients formed the control group, in which standard anti-diabetic therapy was maintained, and 54 patients formed the treatment group, which underwent weekly PIVIT in addition to the administration of standard anti-diabetic therapy. RQ was evaluated monthly in control subjects, and before and after every PIVIT treatment in the treatment group. After weekly PIVIT for 12 weeks, the mean RQ increased from 0.70 to 0.90 in the treatment group, but did not change in the control group. The percentage of subjects reporting adverse events (AEs) was $31.5 \%$ (17/54) in the treatment group and $9.43 \%(5 / 53)$ in the control group $(\mathrm{P}=0.0053)$. The most frequently reported $\mathrm{AE}$ (by 12 subjects) was a gastroenteric reaction when these individuals were receiving $50 \%$ glucose during the PIVIT treatment. The majority of AEs were mild and did not interfere with the ongoing treatment. Thus, PIVIT can be viewed as tolerated and effective for the improvement of RQ in Chinese DM patients. This study was retrospectively registered with the Chinese Clinical Trial
\end{abstract}

Correspondence to: Professor Xiaohui Guo, Department of Endocrinology, Peking University First Hospital, 8 Xishiku Street, Beijing 100034, P.R. China

E-mail: bdyyguoxiaohui@sina.com

Key words: pulsatile intravenous insulin therapy, respiratory quotient, diabetes
Registry (http://www.chictr.org.cn) on November 13th 2019 (registration no. ChiCTR1900027510).

\section{Introduction}

Insulin secretion occurs in a pulsatile manner into the circulation of humans and animals, with fast pulses occurring every 5-15 min $(1,2)$. Insulin is secreted into the portal vein and undergoes partial (40-80\%) hepatic extraction, before being diluted into the systemic insulin pool. Peripheral insulin concentrations oscillate because of the pulsatility of insulin secretions (3). Pulsatile insulin concentrations are important to achieve optimal insulin action, especially the metabolic effects of insulin, such as the suppression of hepatic glucose output and overall insulin secretion (4). However, insulin pulsatility is disrupted in patients with type 2 diabetes mellitus (T2DM) (5), first-degree relatives who lack significant metabolic abnormalities (4), and patients with early type 1 diabetes mellitus (T1DM) (6).

Pulsatile infusion and multiple daily subcutaneous injections have been reported to have a greater hypoglycemic effect than continuous delivery (7), and also to prevent metabolic and microvascular complications in T1DM $(6,8-10)$. Whereas the commonly used methods of therapeutic insulin administration are not pulsatile in nature, several well-known classes of pharmacological agents that are used to treat patients with T2DM increase plasma insulin in a pulsatile manner in both humans and animals, but without significantly modifying insulin pulse frequency $(11,12)$.

Using an artificial pancreas system to replicate the physiological insulin pulse patterns could be one approach to providing effective intravenous insulin infusion in patients with DM for whom traditional therapy is insufficient $(13,14)$. Pulsatile intravenous insulin therapy (PIVIT) involves once-weekly sessions during which pulsatile intravenous insulin and concurrent oral glucose or quantified amounts of carbohydrate are administered, according to a standard protocol and under medical supervision (15).

Respiratory quotient (RQ) is the ratio of the volume of carbon dioxide produced to the volume of oxygen consumed, 
and is an excellent indicator of substrate oxidation (16). Oxygen consumption $\left(\mathrm{VO}_{2}\right)$ and carbon dioxide production $\left(\mathrm{VCO}_{2}\right)$ occur during the oxidation of carbohydrate, protein and fat, but the associated RQ values differ according to the substrate being metabolized.; the RQ values are 0.7 for fat, 0.8 for protein and 1.0 for glucose $(16,17)$. Low RQ has been frequently observed in patients with DM (18), because in diabetes, fat replaces glucose as the main source of energy, and this increase in fat consumption, alongside that of protein, is a contributing factor to hypermetabolism.

The purpose of the present study was to assess the efficacy and safety of PIVIT for the improvement of RQ in Chinese patients with DM.

\section{Subjects and methods}

Ethics statement. The present study was retrospectively registered in the research registry (www.chictr.org.cn; registration no. ChiCTR1900027510; November 13th 2019). The study protocol and informed written consent forms were approved by the research ethics committee of Peking University First Hospital [approval no. (2012)-Instrument Registration No. (15)], and the study was carried out in accordance with the principles of the Declaration of Helsinki. All patients gave their informed written consent to participate in the study.

Study population. The inclusion criteria were a diagnosis of DM that was made according to the 1999 World Health Organization diagnostic criteria, and a measured RQ of $<0.8$. A total of 110 patients with DM were recruited from July 2012 to September 2013 from Peking University First Hospital and Peking University Shougang Hospital, and were enrolled in the present study (Fig. 1). Specific anti-diabetic therapies, including the use of any oral anti-diabetic agents and insulin, were recorded in a questionnaire.

The exclusion criteria were as follows: The presence of hypokalemia; pregnancy or intended pregnancy; or a history of unstable cardiac disease (including new or worsening signs or symptoms of coronary heart disease within 3 months of study entry). Additional exclusion criteria were any of the following within 6 months of study entry: Acute coronary syndrome, stroke or ischemic event; coronary artery intervention or New York Heart Association Class II-IV congestive heart failure; significant renal impairment (creatinine clearance rate $<50 \mathrm{ml} / \mathrm{min}$ ); high ( $>2 \mathrm{X}$ the upper limit of normal) plasma alanine aminotransferase or aspartate aminotransferase activities; or high plasma triglycerides (>600 mg/dl).

The key withdrawal criteria were intolerance of the study drugs, an inability to continue adherence to the protocol and unwillingness to continue in the study.

Randomization. Randomization codes were generated using SAS version 9.10 (SAS Institute, Inc.) for the eligible patients. The researchers randomly assigned cards and ensured that they were sealed inside envelopes with sequence numbers that were the same as the card numbers. Patients were randomly assigned (1:1) to each of the two treatment groups.

Procedures. The total duration of the study was 12 weeks. A total of 53 patients formed the control group. These patients continued their standard anti-diabetic therapy, and had their RQ evaluated every 4 weeks, while 54 patients formed the treatment group (the PIVIT group) and underwent weekly PIVIT in addition to their standard anti-diabetic therapy.

$\mathrm{RQ}$, the ratio of the mean $\mathrm{CO}_{2}$ produced to the mean $\mathrm{O}_{2}$ consumed/minute, was automatically calculated using a VacuMed $^{\circledR}$ (VacuMed YD 17590; Vacumetrics, Inc.). Patients fasted for at least $8 \mathrm{~h}$ overnight, after which indirect calorimetry was performed the following morning. The humidity of the testing room was maintained at $45-60 \%$ and the temperature at $24-26^{\circ} \mathrm{C}$.

The initial PIVIT treatment was carried out three times a day, at 30 min intervals for 2 days, and was then continued once weekly, three times a day. The PIVIT protocol was performed as previously described (6). RQ was evaluated before and after every treatment, and the fasting blood of all patients was collected for the measurement of glucose and lipid levels. Glucose and lipid levels were measured using Beckman Coulter AU chemistry analyzers obtained from commercially available kits (Beckman Coulter, Inc.) according to manufacturer's protocol. A standard questionnaire was completed, which included general information, family history, medical history and details of the medication being used. During the evaluation, height, body mass, blood pressure and pulse were measured. Blood and urine were collected at the baseline stage and after 12 weeks to determine the levels of glucose and glycated hemoglobin (HbA1c), as well as the lipid profile and urinary protein excretion.

The procedures undertaken for the PIVIT group were as follows: i) Capillary blood glucose was measured using an Accu-CHEK ${ }^{\circledR}$ Performa Blood Glucose Meter (Roche Diagnostics). If the blood glucose was $<8 \mathrm{mmol} / \mathrm{l}$, oral glucose $(50 \%$ glucose injection, $20 \mathrm{ml}, 10 \mathrm{~g}$; Otsuka Pharmaceutical Co., Ltd.) was administered to increase the blood concentration. ii) An ambulatory infusion pump (10 pulses/h; Microdose ${ }^{\circledR}$; Bionica Corp.) was used. A volume of $1 \mathrm{ml}$ insulin (Novolin $\mathrm{R}^{\circledR} ; 3 \mathrm{ml} / 300 \mathrm{IU}$; Novo Nordisk A/S) and $9 \mathrm{ml}$ normal saline $(0.9 \%$ saline; Baxter International, Inc.) were mixed in a $10-\mathrm{ml}$ syringe, and the syringe was loaded into the syringe driver on the left of the pump. iii) The infusion pump was then connected to a hand or forearm vein. RQ was evaluated before connecting the pump to a vein. iv) The capillary blood glucose of patients was then monitored every $30 \mathrm{~min}$ by nurses. If blood glucose levels were $<8 \mathrm{mmol} / \mathrm{l}$, patients were administered oral $50 \%$ glucose therapy and their blood glucose concentration was measured every 15 min until it reached $8 \mathrm{mmol} / \mathrm{l}$. v) Resting $\mathrm{VO}_{2}$ and $\mathrm{VCO}_{2}$ were measured after the treatment. vi) The patients left the hospital when they had completed the treatment and their blood glucose was $>8 \mathrm{mmol} / \mathrm{l}$.

Assessment of efficacy. After 12 weeks of treatment, an $\mathrm{RQ} \geq 0.9$ was considered to represent clinical efficacy (6), while an RQ $<0.9$ was considered to represent inefficacy.

Safety assessment. Adverse events (AEs), serious AEs and adverse reactions were recorded during the study period. A serious AE was defined as an event that resulted in death; required inpatient hospitalization or the prolongation of 


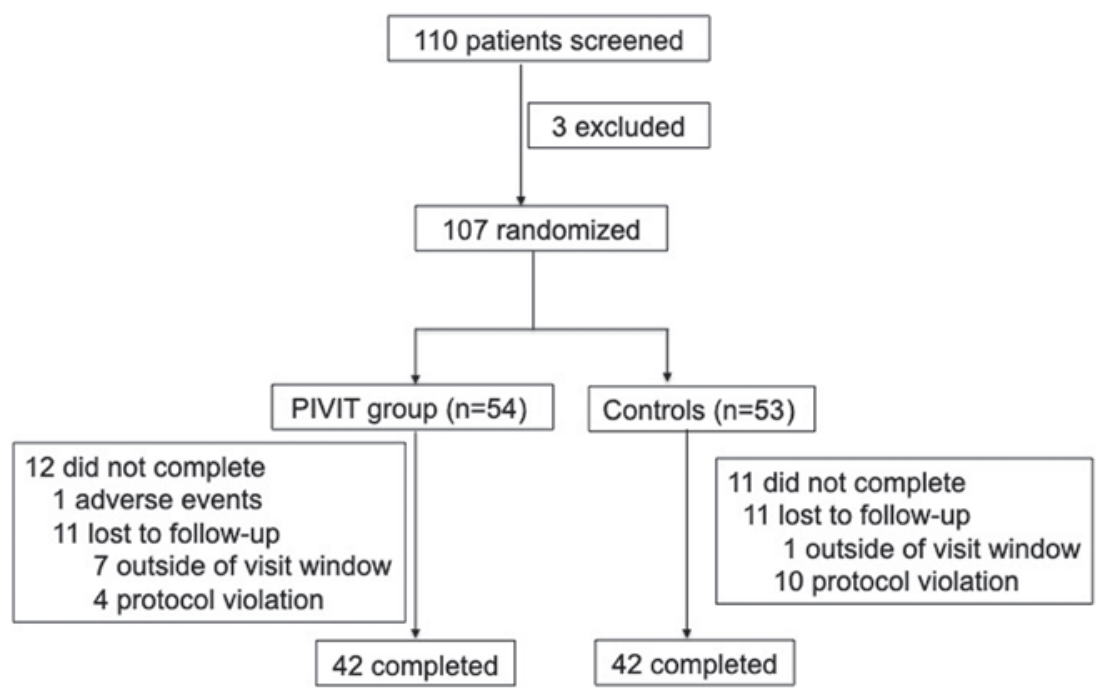

Figure 1. Trial profile. Inclusion and exclusion criteria are presented. The total dropout rate was $21.5 \%$. PIVIT, pulsatile intravenous insulin therapy.

existing hospitalization; resulted in persistent or significant disability/incapacity.

Statistical analysis. All statistical analyses were performed using the SPSS statistical package (version 13.0; SPSS, Inc.). Quantitative data are presented as the mean \pm SD, or the median with minimum, maximum and quartile values. Qualitative data are described as a number and percentage. Paired t-tests were used to analyze changes in clinical parameters from the baseline values. Comparisons of clinical and laboratory parameters between the PIVIT and control groups were performed using paired t-tests or the Wilcoxon rank sum test, as appropriate. The paired-sample t-test was used to compare data before and after treatment. Comparisons of the safety of each therapy were performed using the $\chi^{2}$ test. $\mathrm{P}<0.05$ was considered to indicate a statistically significant difference.

\section{Results}

Baseline clinical characteristics. The baseline clinical characteristics of the subjects are presented in Table I. Compared with treatment group, the control group had a higher proportion of alcohol drinkers and had higher blood glucose concentrations. The age, sex, body mass index, baseline RQ, diabetic complications, blood pressure, respiration rate, heart rate, $\mathrm{HbA1c}$, and anti-diabetic treatments did not differ between the two groups.

Assessment of PIVIT treatment efficacy. Patients in the treatment group showed a significant increase in the RQ after the weekly PIVIT treatment. At the end of the 12-week study period (13 visits), the mean RQ of the PIVIT group had increased from 0.70 to 0.90 , but there was no change in the control group (Table II). The RQ before treatment at each visit was also compared between the treatment and control groups. The RQ at the first visit did not differ between the two groups $(\mathrm{P}=0.273)$, but during the 6, 10 and 13th visits, the RQ before the daily treatment was significantly higher in the treatment than in the control group $(\mathrm{P}<0.05)$ (Table II).

After PIVIT treatment, 26 patients in the treatment group had an $R Q \geq 0.9$, and the clinical efficacy rate was therefore
Proportion of subjects in each group with $R Q \geq 0.9$

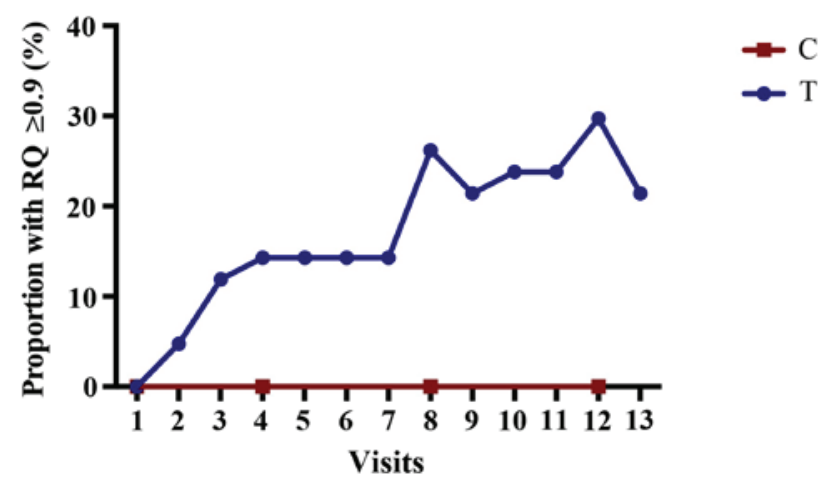

Figure 2. Percentage of subjects in each group with respiratory quotients $\geq 0.9$ before treatment at each visit. C, control group; T, treatment group.

$61.9 \%$ (26/42). By contrast, none of the patients in the control group had an $R Q \geq 0.9$. The proportion of subjects with $\mathrm{RQ} \geq 0.9$ before treatment at each visit is shown in Fig. 2 . In the treatment group, the percentage of subjects with RQ $\geq 0.9$ gradually increased over the study period, peaking at the 12 th visit $(29.7 \%)$, but slightly decreasing at the 13 th visit (to $21.4 \%$ ). However, there was no improvement in the control group.

Assessment of clinical characteristics after PIVIT treatment. The clinical measurement values before and after PIVIT were measured in the treatment group. HbA1c was improved during the study period in subjects in both groups in the present study (PIVIT: $8.02 \pm 1.62$ vs. $7.76 \pm 1.23 \mathrm{mmol} / \mathrm{l}, \mathrm{P}=0.024$; and Control: $7.84 \pm 1.92$ vs. $7.36 \pm 1.30 \mathrm{mmol} / \mathrm{l}, \mathrm{P}=0.003)$. However, there were no significant differences between the two groups after the study period $(\mathrm{P}=0.507$; data not shown). Systolic blood pressure improved $(\mathrm{P}<0.05)$, and blood glucose also showed a non-significant improvement (Table III).

Assessment of the safety of PIVIT. The percentage of subjects reporting AEs was $31.5 \%$ (17/54) in the treatment group and 
Table I. Baseline characteristics of the treatment and control groups.

\begin{tabular}{|c|c|c|c|}
\hline Characteristics & $\mathrm{T}, \mathrm{n}=54$ & $C, n=53$ & P-value \\
\hline Sex, male/female & $32 / 23$ & $37 / 16$ & $0.208^{\mathrm{a}}$ \\
\hline Age, years & $58.52 \pm 11.40$ & $56.14 \pm 10.49$ & $0.264^{\mathrm{b}}$ \\
\hline Height, $\mathrm{m}$ & $1.69 \pm 0.09$ & $1.69 \pm 0.09$ & $0.996^{\mathrm{b}}$ \\
\hline Body mass, $\mathrm{kg}$ & $72.6 \pm 11.79$ & $71.95 \pm 12.51$ & $0.770^{\mathrm{b}}$ \\
\hline BMI, $\mathrm{kg} / \mathrm{m}^{2}$ & $25.39 \pm 3.02$ & $25.17 \pm 3.45$ & $0.718^{\mathrm{b}}$ \\
\hline $\mathrm{VO}_{2}, 1 / \mathrm{min}$ & $0.24 \pm 0.10$ & $0.24 \pm 0.11$ & $0.704^{\mathrm{b}}$ \\
\hline $\mathrm{VCO}_{2}, 1 / \mathrm{min}$ & $0.17 \pm 0.07$ & $0.17 \pm 0.08$ & $0.907^{\mathrm{b}}$ \\
\hline RQ & $0.70 \pm 0.11$ & $0.73 \pm 0.06$ & $0.210^{\mathrm{b}}$ \\
\hline Smoker, yes/no & $19 / 36$ & $22 / 31$ & $0.456^{\mathrm{a}}$ \\
\hline Alcohol drinker, yes/no & $6 / 49$ & $14 / 39$ & $0.038^{\mathrm{a}}$ \\
\hline Diabetic complications, yes/no & $24 / 31$ & $25 / 28$ & $0.712^{\mathrm{a}}$ \\
\hline Oral hypoglycemic agents (\%) & $24(44.4)$ & $21(39.6)$ & $0.613^{\mathrm{a}}$ \\
\hline Insulin treatment $(\%)$ & $13(24.1)$ & $7(13.2)$ & $0.478^{\mathrm{a}}$ \\
\hline Combination of OAD and insulin (\%) & $17(31.5)$ & $25(47.2)$ & $0.203^{\mathrm{a}}$ \\
\hline $\mathrm{SBP}, \mathrm{mmHg}$ & $133.69 \pm 17.37$ & $131.08 \pm 13.71$ & $0.658^{\mathrm{b}}$ \\
\hline $\mathrm{DBP}, \mathrm{mmHg}$ & $77.26 \pm 9.67$ & $80.28 \pm 8.42$ & $0.265^{\mathrm{b}}$ \\
\hline $\mathrm{RR}$, breaths/minute & $17.85 \pm 1.15$ & $17.66 \pm 1.53$ & $0.913^{\mathrm{b}}$ \\
\hline HR, beats/minute & $74.78 \pm 6.98$ & $73.75 \pm 8.60$ & $0.376^{\mathrm{b}}$ \\
\hline Glucose levels, mmol/l & $9.79 \pm 3.18$ & $8.28 \pm 3.22$ & $0.017^{\mathrm{b}}$ \\
\hline $\mathrm{HbA} 1 \mathrm{c}, \%$ & $8.02 \pm 1.62$ & $7.84 \pm 1.92$ & $0.596^{\mathrm{b}}$ \\
\hline
\end{tabular}

Data are presented as the mean \pm SD unless otherwise stated. ${ }^{a}$ Compared using $\chi^{2}$ test; ${ }^{b}$ compared using a t-test. Diabetic complications included diabetic retinopathy, diabetic nephropathy, diabetic neuropathy, and diabetic macrovascular complications. BMI, body mass index; $\mathrm{C}$, control group; DBP, diastolic blood pressure; HR, heart rate; OAD, oral hypoglycemic agents; RQ, respiratory quotient; RR, respiratory rate; SBP, systolic blood pressure; $\mathrm{T}$, treatment group; $\mathrm{VCO}_{2}$, carbon dioxide production; $\mathrm{VO}_{2}$, oxygen consumption; HbA1c, glycated hemoglobin .

Table II. RQ values before and after weekly treatment using PIVIT, compared with control subjects.

\begin{tabular}{|c|c|c|c|c|c|c|c|}
\hline \multirow[b]{2}{*}{ Visit no. } & \multicolumn{3}{|c|}{$\mathrm{T}$} & \multicolumn{2}{|r|}{$\mathrm{C}$} & \multirow[b]{2}{*}{$\mathrm{P}$-value ${ }^{\mathrm{b}, \mathrm{c}}$} & \multirow[b]{2}{*}{ P-value ${ }^{b, d}$} \\
\hline & $\mathrm{n}$ & RQ before treatment & RQ after treatment & $\mathrm{n}$ & $\mathrm{RQ}^{\mathrm{a}}$ & & \\
\hline 1 & 53 & $0.70 \pm 0.11$ & $0.86 \pm 0.08$ & 53 & $0.73 \pm 0.06$ & 0.273 & $<0.0001$ \\
\hline 6 & 50 & $0.79 \pm 0.11$ & $0.89 \pm 0.08$ & 52 & $0.74 \pm 0.07$ & 0.016 & $<0.0001$ \\
\hline 10 & 47 & $0.81 \pm 0.11$ & $0.89 \pm 0.09$ & 52 & $0.75 \pm 0.09$ & 0.007 & $<0.0001$ \\
\hline 13 & 47 & $0.81 \pm 0.09$ & $0.90 \pm 0.08$ & 52 & $0.72 \pm 0.06$ & $<0.0001$ & $<0.0001$ \\
\hline
\end{tabular}

${ }^{\mathrm{a}}$ Evaluated every 4 weeks. ${ }^{\mathrm{b}}$ Comparison made using ANOVA. ${ }^{\mathrm{c}}$ Comparison between RQ before treatment and controls. ${ }^{\mathrm{d}}$ Comparison between RQ after treatment and controls. C, control group; PIVIT, pulsatile intravenous insulin therapy; RQ, respiratory quotient; T, treatment group.

$9.43 \%(5 / 53)$ in the control group $(\mathrm{P}=0.0053)$. No serious AEs occurred in either group. The AEs in the treatment group included 12 cases of gastrointestinal reactions, comprising mainly nausea, vomiting, diarrhea and anorexia, all of which were closely related to taking $10-50 \mathrm{~g}$ of glucose to maintain a blood glucose of $>8 \mathrm{mmol} / 1$ during the treatment, and were relieved by eating or symptomatic treatment. The other adverse reactions are listed in Table IV. Hypoglycemia and gastrointestinal reactions were the most common. Hypoglycemia in the treatment group mostly occurred after treatment or during the evening of the treatment day, whereas hypoglycemia in the control group did not tend to occur at a specific time, and could be rapidly alleviated by eating or consuming oral glucose.

\section{Discussion}

The purpose of the present clinical study was to evaluate the safety and efficacy of PIVIT for the improvement of RQ in patients with T1DM and T2DM, using a repeatable standardized metabolic assay. A total of 110 subjects were enrolled in the present study, of whom 54 were enrolled in the treatment 
Table III. Clinical characteristics of the subjects before and after treatment.

\begin{tabular}{|c|c|c|c|c|c|c|}
\hline \multirow[b]{2}{*}{ Characteristics } & \multicolumn{3}{|c|}{ PIVIT group } & \multicolumn{3}{|c|}{ Control group } \\
\hline & Baseline & Week 12 & P-value ${ }^{a}$ & Baseline & Week 12 & P-value \\
\hline $\mathrm{SBP}, \mathrm{mmHg}$ & $133.69 \pm 17.37$ & $128.00 \pm 16.77$ & 0.028 & $131.08 \pm 13.71$ & $131.65 \pm 14.94$ & 0.82 \\
\hline DBP, mmHg & $77.26 \pm 9.67$ & $75.55 \pm 9.54$ & 0.302 & $80.28 \pm 8.42$ & $79.12 \pm 8.66$ & 0.28 \\
\hline $\mathrm{RR}$, breaths/minute & $17.85 \pm 1.15$ & $17.81 \pm 1.66$ & 0.926 & $17.66 \pm 1.53$ & $17.88 \pm 1.49$ & 0.85 \\
\hline $\mathrm{HR}$, beats/minute & $74.78 \pm 6.98$ & $74.15 \pm 7.62$ & 0.327 & $73.75 \pm 8.6$ & $75.58 \pm 9.87$ & 0.104 \\
\hline Glucose levels, mmol/l & $9.79 \pm 3.18$ & $9.37 \pm 3.69$ & 0.324 & $8.28 \pm 3.22$ & $8.18 \pm 2.83$ & 0.32 \\
\hline $\mathrm{HbA1c}, \%$ & $8.02 \pm 1.62$ & $7.76 \pm 1.23$ & 0.024 & $7.84 \pm 1.92$ & $7.36 \pm 1.3$ & 0.035 \\
\hline
\end{tabular}

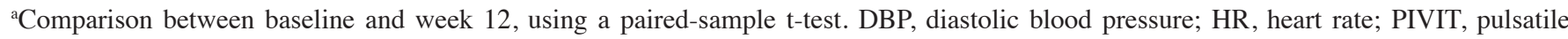
intravenous insulin therapy; SBP, systolic blood pressure; RR, respiratory rate; HbA1c, glycated hemoglobin.

Table IV. AEs in the two groups.

\begin{tabular}{lccc}
\hline AEs & $\mathrm{T}, \mathrm{n}=54$ & $\mathrm{C}, \mathrm{n}=53$ & $\mathrm{P}^{- \text {value }^{\mathrm{a}}}$ \\
\hline Total AEs (\%) & $17 / 54(31.5)$ & $5 / 53(9.43)$ & 0.0053 \\
Gastrointestinal reactions & 12 & 0 & - \\
Hypoglycemia & 7 & 2 & - \\
Fracture & 0 & 1 & - \\
Fever & 1 & 0 & - \\
Fatigue & 2 & 1 & - \\
Chest pain & 0 & 1 & - \\
Orthostatic hypotension & 1 & 0 & - \\
\hline
\end{tabular}

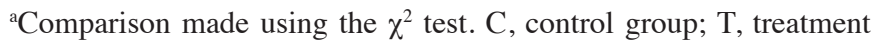
group; AEs, adverse events.

group and 53 in the control group. There were no significant differences in the demographics of the subjects and the baseline data for the two groups were broadly comparable.

RQ is the ratio of $\mathrm{CO}_{2}$ produced to $\mathrm{O}_{2}$ consumed and is helpful in determining the metabolic substrate that is being predominantly catabolized to produce energy $(17,19)$. In resting, post-absorptive non-diabetic individuals, energy requirements are met primarily by fat oxidation, which is reflected in an $\mathrm{RQ}\left(\mathrm{VCO}_{2} / \mathrm{VO}_{2}\right)$ of $0.7-0.8$. After glucose administration, $\mathrm{CO}_{2}$ production (and consequently RQ) increases (to $0.9-1.0$ ), indicating that glucose has become the primary source of energy (20). In theory, RQ may have a value between 0.7 (reflecting 100\% fat oxidation) and 1.0 (reflecting 100\% carbohydrate oxidation). However, healthy adults typically have an RQ of $\sim 0.90$ and patients with DM patients typically have an RQ of 0.7-0.8 $(21,22)$. An impairment in glycogen storage and insulin resistance results in earlier preferential use of fats and proteins as fuel sources. This leads to greater free fatty acid and ketone body production, and a reduction in the RQ. Furthermore, the capacity of patients with DM to oxidize and store exogenous carbohydrate is markedly impaired $(23,24)$, possibly as a result of hyperglycemia (25). Instead, patients with diabetes typically utilize lipid oxidation as their main source of energy $(21,26)$.
In the present study, the changes in RQ during treatment and over the study period were measured and used to evaluate the clinical efficacy of PIVIT. The treatment group underwent PIVIT over 12 weeks, while the subjects in the control group only underwent periodic RQ measurements. RQ gradually increased to $>0.8$ in the treatment group during the early part of the study period, and the mean RQ was $>0.9$ after the 7,8 , 9, 12 and 13th visits. Therefore, PIVIT had a beneficial effect on RQ, especially in the early phase of treatment, although there was a slight deterioration at the final visit. This yielded an efficacy rate was significantly higher than that achieved by the regimen used by the control group, indicating that each PIVIT had a beneficial effect on the RQ.

In previous studies investigating the effects of a combination of weekly outpatient PIVIT and daily subcutaneous insulin treatment, blood pressure was found to improve significantly (27), the progression of diabetic nephropathy slowed $(8,10)$ and diabetic autonomic neuropathy was reversed (9). In the present study, a decrease in systolic blood pressure after treatment was observed. However, the exact mechanism of action behind how PIVIT reduces blood pressure remains to be determined. Because an increase in vascular smooth muscle tone is a hallmark of the hypertensive state in diabetic patients (28), and insulin may play a role in the regulation of vascular smooth muscle (29), it is possible that this therapy partially normalizes vascular reactivity, lowering blood pressure. HbAlc was improved during the study period in subjects in both groups in the present study. This effect of PIVIT may be explained by an increase in the suppression of gluconeogenesis and a consequent reduction in hepatic glucose production (30). However, it may also be that physicians adjusted the therapeutic regimens more frequently, or the patients improved their self-monitoring. An effect of PIVIT on blood glucose, the frequency of complications and blood pressure may require longer periods of study to become apparent.

The incidence of AEs was significantly higher in the PIVIT group than in the control group. The AEs were mild and did not affect the course of treatment. Gastrointestinal reactions included nausea, vomiting, diarrhea, acid reflux, anorexia and a burning sensation in the stomach. All of these occurred in subjects in the treatment group when they were treated with oral high-dose glucose to maintain their blood glucose 
$\geq 8 \mathrm{mmol} / \mathrm{l}$ during PIVIT. Oral high-dose glucose commonly causes gastrointestinal reactions. Previous studies using the $75 \mathrm{~g}$ oral glucose tolerance test (glucose concentration, 25-28.5\%) show a 51.2-66.0\% incidence of gastrointestinal reactions $(31,32)$. Future studies will aim to explore ways to replace high-dose glucose, such as with juice or food.

Hypoglycemia generally occurred after treatment or during the evening of the day of treatment in the PIVIT group. By contrast, hypoglycemia in the control group did not occur at a fixed time and could be rapidly alleviated by eating or consuming oral glucose. Subjects in the treatment group also experienced postural hypotension and cramps in their lower limbs, which were relieved after a break from PIVIT treatment. Hypoglycemia may have been more prevalent in the PIVIT group because the subjects received an intravenous injection of insulin, meaning that they needed to ingest glucose rapidly to avoid this AE. In the early stages of the present study, the lack of timely glucose supplementation at the end of each intervention may have led to this hypoglycemia. The incidence of hypoglycemia was significantly lower in the middle and late phases of the study.

In the present study, PIVIT significantly improved the RQ and blood pressure of diabetic patients. Although the blood glucose concentrations of patients were also slightly improved, this improvement was also demonstrated in the control group, suggesting that the improvement in RQ may be independent of blood glucose concentrations. Previous studies suggest that long-term use of PIVIT delays the progression of diabetic complications $(6,9)$, especially involving the microvascular circulation $(8,10)$. For future studies, the effects of a longer duration of PIVIT treatment on the incidence of the chronic complications of diabetes should be evaluated.

The current study did have limitations. There were no inclusion criteria regarding diabetes duration, medication or blood glucose concentration. Furthermore, no previous studies appeared to identify the effects of diabetes or the degree of glycemic control. However, during the screening period, not all diabetic patients demonstrated a decrease in their RQ values, which may have been because of the duration of their diabetes or better blood glucose control. Patients with a longer disease course and poor blood glucose control should be selected for further studies. Furthermore, patients had to visit a clinic once a week, which may have led to sub-optimal compliance. Additional studies are required to explore effective ways of improving patient compliance.

In conclusion, PIVIT is an effective and tolerated means of improving RQ in Chinese T1DM and T2DM patients.

\section{Acknowledgements}

Not applicable.

\section{Funding}

Beijing Fuji Sunshine Technology Co., Ltd. supported this work.

\section{Availability of data and materials}

The datasets used and/or analyzed during the current study are available from the corresponding author on reasonable request.

\section{Authors' contributions}

NG interpreted the patient data and was a major contributor to the writing of the manuscript. AD, LG and WW interpreted the patient data. $\mathrm{CX}$ and $\mathrm{PH}$ performed the intravenous insulin treatment and RQ measurements. SZ and CY analyzed the patient data. JZ and XG designed the treatment. XG interpreted the data and reviewed the manuscript. All authors read and approved the final manuscript.

\section{Ethics approval and consent to participate}

The study protocol and informed consent forms were approved by the Research Ethics Committee of Peking University First Hospital [approval no. (2012)-Instrument Registration No. (15)], and the study was carried out in accordance with the principles of the Declaration of Helsinki. All patients gave their informed written consent to participate in the study.

\section{Patient consent for publication}

Not applicable.

\section{Competing interests}

The authors declare that they have no competing interests.

\section{References}

1. Satin LS, Butler PC, Ha J and Sherman AS: Pulsatile insulin secretion, impaired glucose tolerance and type 2 diabetes. Mol Aspects Med 42: 61-77, 2015.

2. Lang DA, Matthews DR, Burnett M, Ward GM and Turner RC: Pulsatile, synchronous basal insulin and glucagon secretion in man. Diabetes 31: 22-26, 1982.

3. Pørksen N: The in vivo regulation of pulsatile insulin secretion. Diabetologia 45: 3-20, 2002.

4. Pørksen N, Nyholm B, Veldhuis JD, Butler PC and Schmitz O: In humans at least $75 \%$ of insulin secretion arises from punctuated insulin secretory bursts. Am J Physiol 273: E908-E914, 1997.

5. Scheen AJ, Sturis J, Polonsky KS and Van Cauter E: Alterations in the ultradian oscillations of insulin secretion and plasma glucose in aging. Diabetologia 39: 564-572, 1996.

6. Aoki TT, Benbarka MM, Okimura MC, Arcangeli MA, Walter RM Jr, Wilson LD, Truong MP, Barber AR and Kumagai LF: Long-term intermittent intravenous insulin therapy and type 1 diabetes mellitus. Lancet 342: 515-518, 1993.

7. Matthews DR, Naylor BA, Jones RG, Ward GM and Turner RC: Pulsatile insulin has greater hypoglycemic effect than continuous delivery. Diabetes 32: 617-621, 1983.

8. Weinrauch LA, Burger AJ, Aepfelbacher F, Lee AT, Gleason RE and D'Elia JA: A pilot study to test the effect of pulsatile insulin infusion on cardiovascular mechanisms that might contribute to attenuation of renal compromise in type 1 diabetes mellitus patients with proteinuria. Metabolism 56: 1453-1457, 2007.

9. Weinrauch LA, Bayliss G, Gleason RE, Lee AT and D'Elia JA: Utilization of an abbreviated diabetes impact management scale to assess change in subjective disability during a trial of pulsatile insulin delivery demonstrates benefit. Metabolism 58: 488-491, 2009.

10. Dailey GE, Boden GH, Creech RH, Johnson DG, Gleason RE, Kennedy FP, Weinrauch LA, Weir M and D'Elia J88A: Effects of pulsatile intravenous insulin therapy on the progression of diabetic nephropathy. Metabolism 49: 1491-1495, 2000.

11. Pørksen NK, Munn SR, Steers JL, Schmitz O, Veldhuis JD and Butler PC: Mechanisms of sulfonylurea's stimulation of insulin secretion in vivo: Selective amplification of insulin secretory burst mass. Diabetes 45: 1792-1797, 1996. 
12. Juhl CB, Hollingdal M, Pørksen N, Prange A, Lönnqvist F and Schmitz O: Influence of rosiglitazone treatment on beta-cell function in type 2 diabetes: Evidence of an increased ability of glucose to entrain high-frequency insulin pulsatility. J Clin Endocrinol Metab 88: 3794-3800, 2003.

13. Juhl CB, Gjedsted J, Nielsen MF and Schmitz O: Increased action of pulsatile compared to non-pulsatile insulin delivery during a meal-like glucose exposure simulated by computerized infusion in healthy humans. Metabolism 61: 1177-1181, 2012.

14. Skjaervold NK, Ostling D, Hjelme DR, Spigset O, Lyng O and Aadahl P: Blood glucose control using a novel continuous blood glucose monitor and repetitive intravenous insulin boluses: Exploiting natural insulin pulsatility as a principle for a future artificial pancreas. Int J Endocrinol 2013: 245152, 2013.

15. Aoki TT, Grecu EO, Arcangeli MA, Benbarka MM, Prescott P and Ahn JH: Chronic intermittent intravenous insulin therapy: A new frontier in diabetes therapy. Diabetes Technol Ther 3: $111-123,2001$

16. Livesey $\mathrm{G}$ and Elia M: Estimation of energy expenditure, net carbohydrate utilization, and net fat oxidation and synthesis by indirect calorimetry: Evaluation of errors with special reference to the detailed composition of fuels. Am J Clin Nutr 47: 608-628, 1988.

17. Haugen HA, Chan LN and Li F: Indirect calorimetry: A practical guide for clinicians. Nutr Clin Pract 22: 377-388, 2007.

18. Zhao J, Wang ZY, Li J, Yu HW and Meng QH: Influence of diabetes mellitus on energy metabolism in patients with alcoholic liver cirrhosis. Eur J Gastroenterol Hepatol 32: 110-115, 2020.

19. Graf S, Karsegard VL, Viatte V, Heidegger CP, Fleury Y, Pichard $\mathrm{C}$ and Genton L: Evaluation of three indirect calorimetry devices in mechanically ventilated patients: Which device compares best with the Deltatrac $\mathrm{II}^{\circledR}$ ? A prospective observational study. Clin Nutr 34: 60-65, 2015.

20. Körner A, Eklöf AC, Celsi G and Aperia A: Increased renal metabolism in diabetes. Mechanism and functional implications. Diabetes 43: 629-633, 1994.

21. Gonzalez C, Fagour C, Maury E, Cherifi B, Salandini S, Pierreisnard A, Masquefa-Giraud P, Gin H and Rigalleau V: Early changes in respiratory quotient and resting energy expenditure predict later weight changes in patients treated for poorly controlled type 2 diabetes. Diabetes Metab 40: 299-304, 2014

22. Perseghin G, Lattuada G, De Cobelli F, Esposito A, Costantino F, Canu T, Scifo P, De Taddeo F, Maffi P, Secchi A, et al: Reduced intrahepatic fat content is associated with increased whole-body lipid oxidation in patients with type 1 diabetes. Diabetologia 48 : 2615-2621, 2005.
23. Meyer HU, Curchod B, Maeder E, Pahud P, Jequier E and Felber JP: Modifications of glucose storage and oxidation in nonobese diabetics, measured by continuous indirect calorimetry. Diabetes 29: 752-756, 1980.

24. Thalacker-Mercer AE, Ingram KH, Guo F, Ilkayeva O, Newgard CB and Garvey WT: BMI, RQ, diabetes, and sex affect the relationships between amino acids and clamp measures of insulin action in humans. Diabetes 63: 791-800, 2014

25. Buscemi S, Donatelli M, Grosso G, Vasto S, Galvano F, Costa F, Rosafio $G$ and Verga S: Resting energy expenditure in type 2 diabetic patients and the effect of insulin bolus. Diabetes Res Clin Pract 106: 605-610, 2014.

26. Mirbolooki MR, Taylor GE, Knutzen VK, Scharp DW, Willcourt R and Lakey JR: Pulsatile intravenous insulin therapy: The best practice to reverse diabetes complications? Med Hypotheses 73: 363-369, 2009.

27. Aoki TT, Grecu EO, Prendergast JJ, Arcangeli MA and Meisenheimer R: Effect of chronic intermittent intravenous insulin therapy on antihypertensive medication requirements in IDDM subjects with hypertension and nephropathy. Diabetes Care 18: 1260-1265, 1995.

28. Nieves-Cintrón M, Syed AU, Nystoriak MA and Navedo MF: Regulation of voltage-gated potassium channels in vascular smooth muscle during hypertension and metabolic disorders. Microcirculation 25, 2018.

29. Pietruczuk P, Jain A, Simo-Cheyou ER, Anand-Srivastava MB and Srivastava AK: Protein kinase B/AKT mediates insulin-like growth factor 1-induced phosphorylation and nuclear export of histone deacetylase 5 via NADPH oxidase 4 activation in vascular smooth muscle cells. J Cell Physiol 234: 17337-17350, 2019.

30. Bratusch-Marrain PR, Komjati M and Waldhäusl WK: Efficacy of pulsatile versus continuous insulin administration on hepatic glucose production and glucose utilization in type I diabetic humans. Diabetes 35: 922-926, 1986.

31. Yang H, Wang X, Meng Z, Zhang R, Zhou Y, Zhao Y: Analysis of influence factors of adverse reactions to oral glucose tolerance test in healthy people. J Nur 17: 23-25, 2010.

32. Fan L, Wang G, Tao X, Hong B, Li H, Huang Y: Study on the influencing factors of adverse reactions in 1110 oral glucose tolerance tests. Chin Nur J 29: 387-390, 1994

This work is licensed under a Creative Commons

Attribution-NonCommercial-NoDerivatives 4.0 International (CC BY-NC-ND 4.0) License. 